\section{Mathematical Modeling of Irreversible Electroporation for Treatment Planning}

WWW.tcrt.org

Irreversible Electroporation (IRE) is a new drug-free method to ablate undesirable tissue of particular use in cancer therapy. IRE achieves cell death within the targeted tissue through a series of electric pulses that elevate the transmembrane potentials to an extent that permanently damages the lipid bilayers throughout the treated region. Although the IRE procedure is easy to perform, treatment planning is complicated by the fact that the electric field distribution within the tissue, the greatest single factor controlling the extents of IRE, depends non-trivially on the electrode configuration, pulse parameters and any tissue heterogeneities. To address this difficulty, we instruct on how to properly model IRE and discuss the benefit of modeling in designing treatment protocols. The necessary theoretical basis is introduced and discussed through the detailed analysis of two classic dual-electrode configurations from electrochemotherapy: coaxial disk electrodes and parallel needle electrodes. Dimensionless figures for these cases are also provided that allow cell constants, treated areas, and the details of heating to be determined for a wide range of conditions, for uniform tissues, simply by plugging in the appropriate physical property values and pulse parameters such as electrode spacing, size, and pulse amplitude. Complexities, such as heterogeneous tissues and changes in conductivity due to electroporation, are also discussed. The synthesis of these details can be used directly by surgeons in treatment planning. Irreversible electroporation is a promising new technique to treat cancer in a targeted manner without the use of drugs; however, it does require a detailed understanding of how electric currents flow within biological tissues. By providing the understanding and tools necessary to design an IRE protocol, this study seeks to facilitate the translation of this new and exciting cancer therapy into clinical practice.

Key words: Tumor ablation; Complete regression; Electric pulses; Electropermeabilization; Pulsed electric fields (PEFs); and Cancer therapy.

\section{Introduction}

Irreversible electroporation (IRE) is a new minimally invasive surgical technique to ablate undesirable tissue (1). The technique is easy to apply, can be monitored and controlled, is not affected by local blood flow, and does not require the use of adjuvant drugs. The procedure involves placing electrodes into or around the targeted area to deliver a series of short and intense electric pulses that induce irreversible structural changes in the cell membranes of the targeted tissue through a phenomenon known as irreversible electroporation $(2,3)$, causing cell death.

Electroporation, which results in an increase in the permeabilization of the cell membrane, can be initiated by exposing cells or tissues to electric pulses $(4,5)$.
Jon F. Edd ${ }^{1}$

Rafael V. Davalos,",

${ }^{1}$ Center for Engineering in Medicine

Massachusetts General Hospital

Harvard Medical School and Shriners

Hospital for Children

Boston, MA 02114, USA

${ }^{2}$ School of Biomedical

Engineering and Sciences

Virginia Tech - Wake Forest University

Blacksburg, VA 24061, USA
${ }^{*}$ Corresponding Author: Rafael V. Davalos, Ph.D. Email: davalos@vt.edu 
As a function of the resulting transmembrane potential (the electric potential difference across the plasma membrane) the electroporation pulse can either: have no effect on the cell membrane, reversibly open the cell membrane after which cells can survive, or irreversibly open the cell membrane, which leads to cell death. This potential is normally on the order of $70 \mathrm{mV}$. If the potential drop across the membrane exceeds approximately $1 \mathrm{~V}$, structural rearrangement of the lipid bilayer occurs, creating permanent aqueous pathways or pores for ions and macromolecules to pass through, i.e., irreversible electroporation (6).

This induced potential is dependent on a variety of conditions such as tissue type and cell size. There are also a number of pulse parameters that affect the resulting transmembrane potential and, thus, the degree of electroporation, such as the pulse shape, duration, number, and repetition rate. Readers interested in electroporation at the single cell level are referred to (7-10). Weaver et al. illustrate how an applied electric field in tissue affects the transmembrane potential of its cells and the equivalent circuit of tissue undergoing electroporation (11). The microscopic electric field distribution is difficult to determine analytically due to tissue anisotropy, the locations of large blood vessels, electrode geometry and orientations, and the distribution of electrical impedance within the tissue. The issue is further complicated because the bioelectrical properties will change due to minor localized heating of the tissue from the pulses and due to the cells becoming permeable as a result of treatment $(12,13)$. However, for a specific tissue type, the macroscopic electric field distribution to which the tissue is exposed is the primary parameter (14). Therefore, knowledge of the electric field distribution is critical in optimizing pulse parameters for in vivo applications.

Reversible electroporation of tissue has been used in conjunction with membrane impermeant anticancer drugs for almost twenty years as a form of cancer therapy known as electrochemotherapy (ECT) (15-17). Once the non-permeable substance is injected into the body, electrodes placed into or around the targeted tissue generate reversibly permeabilizing electric fields throughout the targeted tissue to facilitate the entry of the drug into the cells of the targeted area (18) to enhance the effectiveness of the anticancer drug. The cell membrane subsequently reseals itself with the drug trapped inside the cell. Typical parameters for electrochemotherapy are 4-8 $100 \mu$ s pulses at a frequency of $1 \mathrm{~Hz}$ with a voltage-todistance ratio of $1300 \mathrm{~V} / \mathrm{cm}$ (19). ECT benefits from the ease of application and the area treated is comparable to both high temperature treatment therapies and non-selective chemical therapies. In addition, because the electric field is not strongly affected by local blood flow, control over the extent of the affected tissue is possible, unlike in many other diffuse modes such as thermal and non-selective chemical therapies. ECT is beneficial due to its selectivity; however, a disadvantage is that by its nature, it requires the combination of chemical agents with an electric field to have the desired effect.

Researchers in electrochemotherapy have demonstrated the importance of the electric field distribution to induce reversible electroporation to enhance the effectiveness of drug delivery (20). Miklavcic et al. (1998) recognized the importance of the electric field distribution when designing electroporation protocols and showed that minimally invasive needle electrodes produce an inherently inhomogeneous field that must be taken into consideration when planning a treatment. By comparing their models with experimental data, they have found that for a specific tissue type and a specified set of voltage parameters, there is a strong correlation between the electric field and the tissue's permeabilization state (21). They estimated the electric field threshold for reversible electroporation as $362+/-21 \mathrm{~V} / \mathrm{cm}$ and the threshold for irreversible electroporation as $637+/-43 \mathrm{~V} / \mathrm{cm}$ for rat liver tissue using eight $100 \mu$ s pulses at a frequency of $1 \mathrm{~Hz}$. Such studies are valuable for determining the electric field thresholds to induce reversible and irreversible electroporation for a specific tissue and set of voltage parameters. The culmination of the research by Miklavcic et al. illustrates the effectiveness of modeling to select appropriate pulse parameters and electrode geometries to successfully treat a targeted area for ECT (21).

Recently, Davalos et al. developed models to study the potential thermal effects associated with reversible electroporation. They analytically investigated the effects of various parameters on the resultant temperature distribution resulting from reversible electroporation pulses (22). They have shown what parameters are optimal to ensure negligible heating of the tissue. For example, electrodes with smaller diameters result in higher and less uniform distribution of electric fields. Other researchers showed that the temperature increase and the thermal damage is typically insignificant unless the procedure is conducted over the skin $(23,24)$. When designing protocols for transdermal drug delivery via electroporation, they showed that it is important to recognize that a large amount of the voltage drop occurs over the stratum corneum $(25,26)$. When the electrodes are placed over the skin, significant heating can occur due to the high electrical resistance of the skin, unless active cooling is applied (23). Furthermore, improperly prepared skin can drastically reduce the actual electric field delivered within the tissue when electrodes are applied at the body surface.

Similarly, in order to ablate only the targeted tissue with irreversible electroporation, there are specific parameters that must be chosen. Through modeling and simulations, one can accurately predict the necessary electrode voltage as well as optimize the electrode placements, pulse patterns, timing, et cetera, for each unique treatment to effectively treat the targeted area. Previous models have shown that IRE can ab- 
late substantial volumes of tissue without inducing any relevant thermal effects (1-3). The research showed that the most critical parameters affecting the extents of ablation are impedance distribution, pulse characteristics, and electrode configuration (size, spacing, and number) (1).

The primary goal of this paper is to provide interested physicians and researchers with the tools and understanding necessary to design appropriate protocols to use of IRE in cancer therapy. To this end, we first present the fundamental theory that determines how electric fields will result from a chosen electrode configuration, pulse characteristics, and the electrical properties of the tissue. We then proceed to describe the other important theoretical paradigm for modeling IRE, heat diffusion via the Pennes bioheat equation, and we detail the connection between them, namely Joule heating.

To provide an intuitive understanding of how to design an effective IRE procedure, we then proceed to discuss in detail two important cases that come up frequently in IRE treatments: coaxial disk electrodes and parallel needle electrodes. To expound upon these fundamental 2-electrode cases across a range of relevant electrode sizes and interelectrode gaps, we provide the reader with a functional set of figures into which they can plug their scenario specific parameters. We then discuss the use of cell constants to predict power consumption and measure bulk conductivity, explain how to predict and control the size of the treated region, and provide the tools necessary to estimate the extents, if any, of thermal damage at various points within the treated tissue. We also discuss various complexities of IRE such as heterogeneous tissues and the changes in tissue conductivity that result from electroporation.

The synthesis of these various aspects will provide physicians with a framework to design IRE protocols for their particular application. If the physician knows the electrode configuration and geometry as well as the electrical impedance distribution of the tissue, pulse parameters can be designed to ensure ablation with irreversible electroporation throughout the entire targeted region.

\section{Materials and Methods}

In order to design protocols for an IRE procedure, the electric field distribution must be determined, which is dependent on the procedure's specific electrode geometry and tissue impedance distribution. By predicting the electric field distribution for a specific scenario, the physician can optimize the electrode geometry to ablate the entire targeted region while minimally affecting the surrounding tissue. Furthermore, to verify that a specific protocol does not induce thermal effects, the temperature distribution can be calculated from the electric field distribution, the electric pulse parameters and tissue properties. The methodology below illustrates how to obtain this information and presents the results through two basic two-dimensional models: coaxial disk electrodes and parallel needle electrodes.

\section{Estimating the Electric Field Distribution \\ Due to Electroporation}

The electrical field distribution associated with an electroporation pulse is determined by solving the Laplace equation:

$$
\nabla \cdot(\sigma \nabla \phi)=0
$$

where $\phi$ is the electrical potential and $\sigma$ is the electrical conductivity of the tissue. The solution of the Laplace equation yields the distribution of the potential within the tissue. The electric field is then the gradient of the potential, i.e., $E=\nabla \phi$.

The typical formula to approximate the induced transmembrane potential $\left(\mathrm{V}_{\mathrm{m}}\right)$ resulting from an applied electric field, which determines whether a cell will undergo electroporation, is:

$$
V_{m}=\lambda r E_{a} \cos (\theta) \cdot\left[1+\left(f / f_{s}\right)^{2}\right]^{-0.5}
$$

where $\lambda$ is the shape factor of the cell (1.5 for spherical cells), $r$ is the radius of the cell, $E_{a}$ is the applied electric field, $\theta$ is the angle between electric field and the vector from the cell center to any point on its surface, $f_{s}$ is approximately equal to the frequency where the beta dielectric dispersion occurs (below which the cell membrane charge is in step with the electric field), and $f$ is the frequency of the assumed sinusoidal $E_{a}(27)$. Since even a DC pulse can be decomposed into a superposition of various sine waves at different frequencies, this equation enables the transmembrane potential resulting from a short pulse to be computed in an approximate sense.

For most cases, the transient terms can be neglected because the electroporation pulse ( $100 \mu \mathrm{s}-50 \mathrm{~ms})$ is much larger than the membrane charging time (about $1 \mu \mathrm{s}$ ) (28). The equation above reveals that if the pulses are ultrashort (i.e., less than several microseconds), the problem is no longer static and the charging time of the membrane must be considered. Under such circumstances, it is hypothesized that the lipid bilayer membrane is not electroporated but that intracellular organelles are affected by the pulsed electric field (29).

\section{Estimating the Temperature Distribution Due to Electroporation}

Solving the Laplace equation enables one to calculate the associated Joule heating ( $\mathrm{p})$, the heat generation rate per unit volume from an electric field: 


$$
p=\sigma|\nabla \phi|^{2}
$$

The heating of the tissue resulting from each procedure was calculated by adding the Joule heating source term to the Pennes Bioheat transfer equation (30). The Pennes Bioheat equation, which accounts for metabolism and blood flow, is the most commonly used equation to solve heat transfer problems in the body. Our modified Pennes Bioheat equation has the following form:

$$
\nabla \cdot(k \nabla T)+w_{b} c_{b}\left(T_{a}-T\right)+q^{\prime \prime \prime}+p=\rho c_{p} \frac{\partial T}{\partial t},
$$

where $k$ is the thermal conductivity of the tissue, $T$ is the temperature, $w_{b}$ is the blood perfusion, $c_{b}$ is the heat capacity of the blood, $T_{a}$ is the arterial temperature, $\mathrm{q}^{\prime \prime \prime}$ is the metabolic heat generation, $\rho$ is the tissue density, and $c_{p}$ is the heat capacity of the tissue.

For software that cannot handle the blood perfusion term in Equation [4], a variable can be substituted to circumvent this limitation. Let:

$$
T-T_{a}=U e^{\frac{-w_{b} c_{b} t}{\rho c_{p}}} .
$$

Therefore, the transformation is:

$$
\begin{array}{r}
(\nabla \cdot k \nabla U) e^{\frac{-w_{b} c_{b}}{\rho c_{p}} t}-w_{b} c_{b} U e^{\frac{-w_{b} c_{b}}{\rho c_{p}} t}+\left(q^{\prime \prime \prime}+p\right)= \\
\rho c_{p} \frac{\partial U}{\partial t} e^{\frac{-w_{b} c_{b}}{\rho c_{p}} t}-\rho c_{p} \frac{-w_{b} c_{b}}{\rho c_{p}} U e^{\frac{-w_{b} c_{b}}{\rho c_{p}} t} .
\end{array}
$$

After canceling out like-terms and dividing through by the exponential, the equation reduces to:

$$
(\nabla \cdot k \nabla U)+\left(q^{\prime \prime \prime}+p\right) e^{\frac{-w_{b} c_{b}}{\rho c_{p}} t}=\rho c_{p} \frac{\partial U}{\partial t} .
$$

The equation above resembles the general heat conduction equation, which is readily solvable with most finite element packages, with the term as the heat source $\left(q^{\prime \prime \prime}+p\right) e^{\frac{-w_{b} c_{b}}{\rho c_{p}} t}$.

The same variable substitution is applied to the boundary conditions to solve the problem. The solution, $\mathrm{U}$, is then substituted back into [5] to determine the temperature distribution in the analyzed domain.

\section{Case Studies}

Two fundamental models that are representative of common conditions associated with irreversible electroporation are used to illustrate the critical parameters in designing treatments. These models are depicted in Figure 1. The first configuration involves two coaxial disk electrodes and the second contains two parallel needle electrodes. These are common electrode geometries and many more geometries are of course possible, yet through these two basic examples, we illustrate the trends that occur as a function of critical parameters such as electrode size and shape.

The models used to generate the results in Figures 3, 4, and 6 are two-dimensional, as depicted in Figure 2. For the disk electrodes, as long as conductivity is uniform, no loss of generality is incurred by using a cylindrically symmetric model. However, the $2 \mathrm{D}$ simplification for the needle electrodes implies infinitely long electrodes. As will be discussed in the following sections, this causes the cell constant, which can be used to predict power consumption or to estimate bulk tissue conductivity, to be overestimated, the treatment volume to be underestimated, and has minimal impact on the predicted maximal temperature rise due to the pulse. These errors become substantial when the ratio of electrode length (L) to electrode gap (d) is small, yet it will be shown that the 2D model is still a useful approximation for the purpose of IRE treatment planning.

\section{Boundary Conditions}

The models are fully defined and readily solvable using a numerical method once an appropriate set of boundary conditions and the properties of the tissue are defined. Boundary conditions most often include surfaces where electric potential is specified, as in the case of a source or sink electrode, or surfaces that are electrically insulating, as on the free surfaces of the tissue, for example. For each electrode configuration, the surface of one electrode is assumed to have a prescribed voltage, and the other electrode is set to ground. Specifically, the boundary condition of the tissue that is in contact with a charged electrode is defined as:

$$
\phi=V_{o}
$$

where $V_{o}$ is the applied voltage and the electrical boundary condition at the interface of a grounded electrode and the tissue is defined as:

$$
\phi=0
$$

The boundaries where the analyzed domain is not in contact with an electrode are treated as electrically insulating:

$$
\frac{\partial \phi}{\partial n}=0 .
$$

Other possibilities for electrode boundary conditions include those for passive electrodes, where a film conductance is specified, or those where the electrode interface impedance is included in an approximate manner, as in the complete electrode model (31). 
The computation of $\phi$ and, hence, the electric field can then be used to estimate the variation of temperature, where the tissue is initially at physiological temperature $\left(37^{\circ} \mathrm{C}\right)$. Several thermal boundary conditions can be explored to study their heat exchange between the electrodes and the tissue (1, $2,22,24)$; however, in our study, the boundary of the analyzed domain and the surfaces of the electrodes are taken to be adiabatic to produce an upper limit to the calculated temperature distribution in the tissue:

$$
\frac{\partial T}{\partial n}=0
$$

\section{Numerical Modeling}

In this study, the computations were performed with a commercial finite element package (FEMLab, Comsol AS, Stockholm, Sweden), whereas data analysis was done in Matlab (The MathWorks, Inc., Natick, MA). The finite element method generates approximate solutions that consist of the value of electric potential on every node within a mesh that fills the geometric confines of the tissue in question. Analytical solution of electrical conduction on a non-trivial geometry usually requires the use of techniques of numerical analysis, such as the finite difference method or the finite element method. Even where analytical solutions exist, it is often difficult to extract accurate predictions due to the frequent occurrence of infinite integrals that are difficult to evaluate (even numerically). Furthermore, analytical solutions typically fail when the tissue conductivity is heterogeneous. In our study, the analyzed domain extends far enough (typically at least ten times the electrode gap for the case of needle electrodes and five times for disk electrodes) from the area of interest (i.e., near the electrodes) that the electrically and thermally insulating boundary at the edges of the domain does not significantly influence the results in the treatment zone.

\section{Results and Discussion}

\section{Example of Electric Field Distribution for Each Electrode Configuration}

Figure 1 depicts the electric field that was computed for the two cases with a scalar and uniform electrical conductivity across the tissue, and Figure 2 displays six additional representative cases in 2D. The first case (Fig. 1a) was computed for a pair of coaxial disk electrodes with an electrode-electrode gap (d) of $4 \mathrm{~mm}$ and a disk diameter (D) of $10 \mathrm{~mm}$, and where a pulse of $400 \mathrm{~V}$ was applied across the electrodes. The second case (Fig. 1b) was computed for a pair of 1-mm diameter (D) parallel needle electrodes with a center-to-center distance (d) of $10 \mathrm{~mm}$, and a pulse of $1000 \mathrm{~V}$ was applied between the two needles. In each case, the voltageto-distance ratio is $1000 \mathrm{~V} / \mathrm{cm}$, but it is important to notice that the actual electric field that develops in the area between the electrodes varies strongly with position, especially in the case of needle electrodes. In fact, the regions with the largest electric fields $(1600 \mathrm{~V} / \mathrm{cm})$ occur as the pair of thin red annular rings in the first case and the thin red covering over the needle electrodes in the second case. These high fields are due to the sharp electrode borders in the first case and the electrode geometry in the second case. It is also apparent from the cases reported in Figure 2 that electrode diameter greatly affects the region of tissue that will experience any particular electric field strength (larger electrodes mean larger treatment volumes if all other parameters are fixed).

The results presented in Figures 1 and 2 are for a specific set of conditions. Figures 3, 4, and 6 provide vital statistics and trends for the possible variations on these two basic designs.

\section{Cell Constants, Power Consumption, and Bulk Conductivity}

The cell constant $(\mathrm{K})$ is numerically equal to the product of the bulk conductivity (in $\mathrm{S} / \mathrm{m}$ ) of the tissue $(\sigma)$ and the magnitude of electrical impedance ( $\mathrm{Z}$, in Ohms) between the electrodes, and has units of inverse length. Figure 3 presents the cell constants $(\mathrm{K})$ computed for the two electrode configurations over a range of $d / \mathrm{D}$ values typically encountered. To obtain these data, the applied current was calculated by integrating the normal current density over the surface of one electrode. Most importantly, $K$ provides: (i) an estimate of $\sigma$ when $Z$ has been measured and (ii) an estimate of the power consumption when $\sigma$ is known ahead of time $\left(\mathrm{P}=\sigma \mathrm{V}^{2} / \mathrm{K}\right)$.

For example, consider the two sets of cases presented in Figure 2. For the disk electrode case where $d / D=0.4$ (Fig. 2c), $K D$ $\approx 0.37\left(\mathrm{~K}=37 \mathrm{~m}^{-1}\right)$ from Figure 3a. Similarly, for the needle electrode case where $d / D=10$ (Fig. $2 \mathrm{e}$ ), $K L \approx 0.96$ ( $K=96$ $\mathrm{m}^{-1}$ for $\left.L=10 \mathrm{~mm}\right)$. If we assume $\sigma \approx 0.2 \mathrm{~S} / \mathrm{m}$, then the power during the pulse will be around $0.86 \mathrm{~kW}\left(\mathrm{P} \approx 0.2 \cdot 400^{2} / 37\right)$ for the disk electrode case in Figure $2 \mathrm{c}$ and about $2.1 \mathrm{~kW}(\mathrm{P} \approx$ $0.2 \cdot 1000^{2} / 96$ ) for the needle electrode case in Figure 2e. For the needle electrode configuration, however, this $2 \mathrm{D}$ simplification has resulted in a lower than actual rate of power consumption during the pulse being predicted. The full 3D model predicts that the pulse will in fact consume $2.8 \mathrm{~kW}$, so that $K$ $=72 \mathrm{~m}^{-1}$. This discrepancy can be understood by considering that the impedance in the circuit falls when current can flow not only within the $10 \mathrm{~mm}$ thick section of tissue that bounds the electrodes, but also above and below it; since adding impedances in parallel will reduce the overall impedance $(Z)$, the $3 \mathrm{D}$ model will always have higher power consumption and a lower cell constant than would be predicted from the $2 \mathrm{D}$ model. This effect becomes more pronounced as $L / d$ is reduced.

Table I examines this discrepancy between the cell constant predicted for the case of $1 \mathrm{~mm}$ diameter parallel needle electrodes by the 2D model and that for the full 3D model. 
(a)

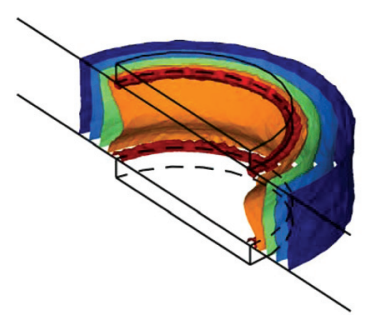

(b)

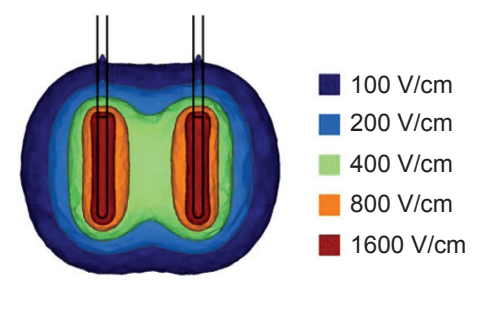

Figure 1: Two commonly employed electrode designs - (a) coaxial disk electrodes and (b) parallel needle electrodes. Full 3D solution is depicted for each case, where the aspect ratio $(\mathrm{d} / \mathrm{D}=$ interelectrode gap/electrode diameter) is 0.4 for (a) and 10 for (b), and where the applied voltage to distance ratio $(\mathrm{V} / \mathrm{d})$ is $1000 \mathrm{~V} / \mathrm{cm}$.

(a)

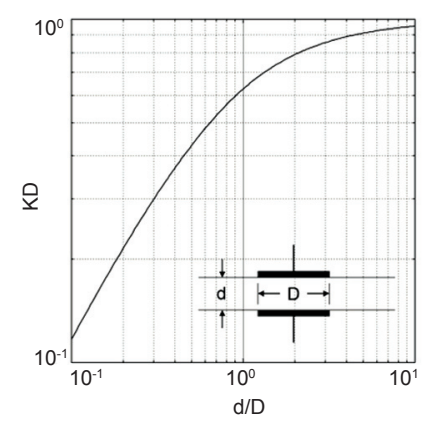

(b)

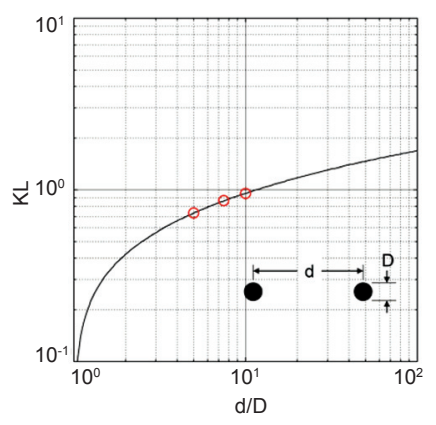

Figure 3: Cell constant (K) as a function of aspect ratio (d/D) plotted for (a) coaxial disk electrodes and (b) parallel needle electrodes. Cell constant is non-dimensionalized in the first case by multiplying with the diameter (D) of the disk electrodes and in the second case by multiplying with the active electrode length normal to the page (L). Circles in (b) correspond with the three columns in Table I that report discrepancies between the 2D assumption (infinitely long parallel needle electrodes) and the fully 3D case.

(a)

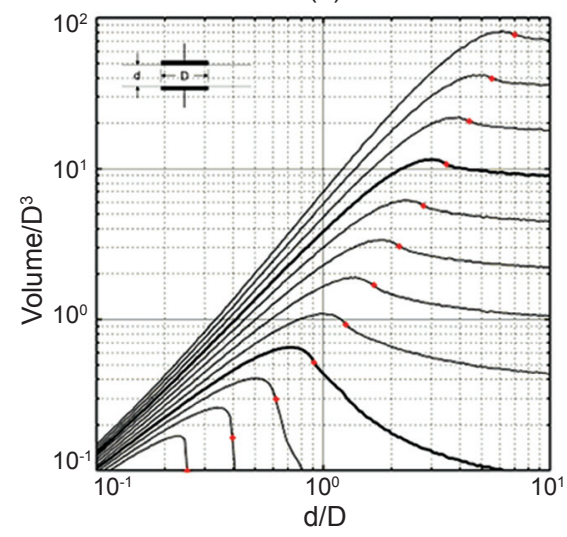

(a)

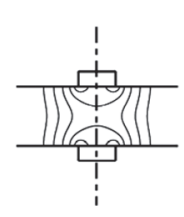

(d)

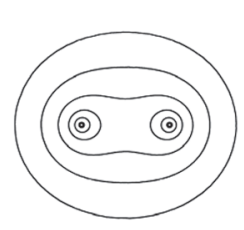

(b)

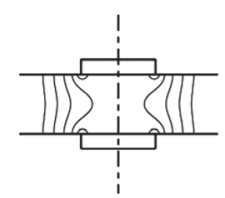

(e)

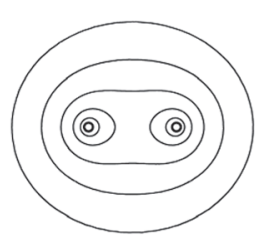

(c)

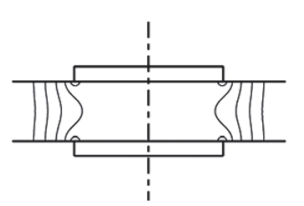

(f)

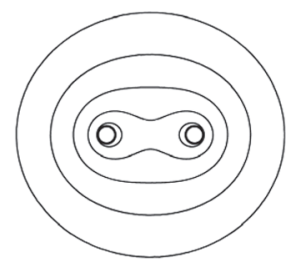

Figure 2: Cross-sectional electric field distribution for some representative cases. Graphs show simplified 2D models where the same surfaces of constant electric field from Figure 1 are represented with contours. Electrode diameters are 2.5, 5, and $10 \mathrm{~mm}$ for (a)-(c) and $0.5,1$, and $2 \mathrm{~mm}$ for (d)-(f), respectively. All other parameters are identical with those listed for the corresponding graphs of Figure 1, so that (c) and (e) are for the same two cases as shown in Figure 1. For the disk electrodes in (a)-(c), cylindrical symmetry ensures the accuracy of the 2D model; however, the 2D simplification for the needle electrodes in (d)-(f), where the electrodes are perpendicular to the plane of the model, implies infinitely long electrodes.

From these computed data, it can indeed be seen that as $L$ increases for constant $d$, the 2D model becomes more accurate. Conversely, the 2D model becomes less accurate as $d$ is increased across the three columns that correspond with the $d / D$ points marked as circles in Figure $3 b$. By adjusting the data in Figure $3 \mathrm{~b}$ according to the trends in Table I, it is possible to generate reasonably accurate predictions of the

(b)

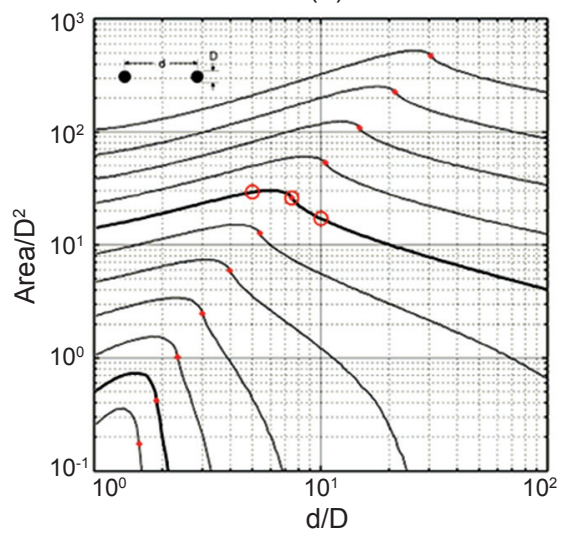

Figure 4: Size of the "treated" region as a function of aspect ratio (d/D). (a) Volume (normalized by $\mathrm{D}^{3}$ ) of the revolved cross sectional area within a coaxial disk configuration where electric field strength (normalized by V/D) exceeds, from the top to the bottom curve, $10^{-1.6}, 10^{-1.4}, \ldots, 10^{0.6}$. (b) Cross sectional area (normalized by $\mathrm{D}^{2}$ ) within the parallel needle electrodes configuration where the similarly normalized electric field strength exceeds, from the top to the bottom curve, $10^{-1.8}, 10^{-1.6}, \ldots$, $10^{0.2}$. Bold curves are for 0.1 and 1 in both graphs. The asterisk on each curve denotes the point where the region containing electric field strengths above a certain level (different for each curve) splits in two, or in other words where the particular level set (in normalized electric field strength) passes through the central point in the geometry. Circles in (b) along the curve $E /(V / D)=0.1$ correspond with the three columns in Table II that report discrepancies between the 2D assumption (infinitely long parallel needle electrodes) and the fully 3D case.

cell constant with just the 2D model.

\section{Controlling the Size of the Treated Region}

The data presented in Figure 4 allows the reader to optimize treatment area for most situations with these two types of electrodes. In this figure, the volume for disk electrodes, and cross-sectional area for needle electrodes, of tissue with electric field strengths above certain levels is plotted across a relevant range of $d / D$ values. After looking at the data, which are presented in dimensionless form, some trends become apparent. In both systems, small electric field strengths exist over the widest regions, and large electric field strengths are more confined in space. Additionally, the treated region gradually increases in size as the electrodes are pulled apart from the closest separation, until just before the point where the treated region splits in two. 
Table I

Cell constant discrepancy $\left(K_{3 \mathrm{D}} / K_{2 \mathrm{D}}\right)$

\begin{tabular}{lccc}
\hline $\mathrm{d}=$ & $5 \mathrm{~mm}$ & $7.5 \mathrm{~mm}$ & $10 \mathrm{~mm}$ \\
\hline $\mathrm{L}=2.5 \mathrm{~mm}$ & 0.51 & 0.47 & 0.45 \\
$\mathrm{~L}=5 \mathrm{~mm}$ & 0.65 & 0.61 & 0.57 \\
$\mathrm{~L}=10 \mathrm{~mm}$ & 0.78 & 0.74 & 0.71 \\
$\mathrm{~L}=20 \mathrm{~mm}$ & 0.86 & 0.84 & 0.81 \\
\hline
\end{tabular}

After that point, the treated zone shrinks rapidly on further separation of the electrodes. These plots reveal how large the treated region will be and what the distance between two electrodes of fixed diameter (D) should be to maximize the size of the treated region resulting from a particular pulse amplitude (V) applied between the electrodes.

(a)

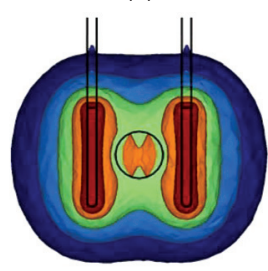

(b)

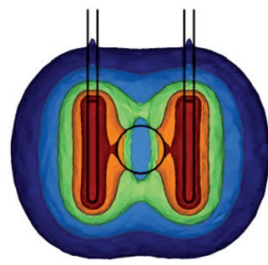

(c)

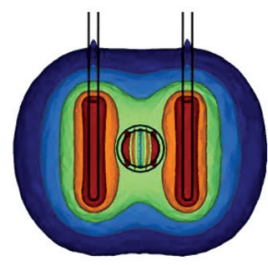

Figure 5: Effect of a heterogeneous tissue conductivity on the electric field distribution. Each graph depicts the surfaces of constant electric field strength (for 100, 200, 400, 800, and $1600 \mathrm{~V} / \mathrm{cm}$ ) that would result from the presence of a $5 \mathrm{~mm}$ diameter spherical inclusion, located halfway between the electrodes. The inclusion is composed of tissue with one fifth or five times the background electrical conductivity in graphs (a) and (b), respectively, while (c) the third graph is similar to (a) but with a nested $4 \mathrm{~mm}$ diameter sphere of five times normal conductivity. Color map, electrode geometry, pulse amplitude, and background tissue properties are identical to the similar 3D model of needle electrodes in Figure 1b.

Table II

Treated volume discrepancy $\left(V_{3 \mathrm{D}} / V_{2 \mathrm{D}}\right)$

\begin{tabular}{cccc}
\hline \multicolumn{1}{c}{$\mathrm{d}=$} & $5 \mathrm{~mm}$ & $7.5 \mathrm{~mm}$ & $10 \mathrm{~mm}$ \\
\hline $\mathrm{L}=2.5 \mathrm{~mm}$ & 1.44 & 1.33 & 1.86 \\
$\mathrm{~L}=5 \mathrm{~mm}$ & 1.25 & 1.20 & 1.61 \\
$\mathrm{~L}=10 \mathrm{~mm}$ & 1.15 & 1.10 & 1.41 \\
$\mathrm{~L}=20 \mathrm{~mm}$ & 1.08 & 1.08 & 1.24 \\
\hline
\end{tabular}

For example, consider the disk electrode case from Figure 2c, where $d / D=0.4$ and $V / D=400 \mathrm{~V} / \mathrm{cm}$ and assume that the IRE threshold is $800 \mathrm{~V} / \mathrm{cm}$. The volume of tissue that will experience electric fields in excess of $800 \mathrm{~V} / \mathrm{cm}$ can be estimated by locating approximately where the curve $E /(V / D)=2$ or $10^{0.3}$ exists. Since there is no curve where $E /(V / D)$ is exactly $10^{0.3}$, we must interpolate between the closest neighboring curves, which are the third $\left(10^{0.2}\right)$ and second $\left(10^{0.4}\right)$ curves from the bottom. Subsequently, locate the y-coordinate on the appropriate curve of constant $E /(V / D)$ in Figure 4a, where the $\mathrm{x}$-coordinate $(\mathrm{d} / \mathrm{D})$ is 0.4 . From this we can see that the appropriate coordinates on Figure $4 \mathrm{a}$ would be $(\mathrm{x}=0.4, \mathrm{y} \approx$ 0.3 ). Using this information we can approximate the volume of treatment $\left(\mathrm{Vol} / \mathrm{D}^{3} \approx 0.3\right)$ as $0.3 \mathrm{~cm}^{3}$. This matches our expectations from the extents of the $800 \mathrm{~V} / \mathrm{cm}$ contour visible in Figure 2c, which is nearly equivalent to the tissue directly between the $10 \mathrm{~mm}$ diameter electrodes $\left(0.314 \mathrm{~cm}^{3}\right)$. It is also interesting to note that the inter-electrode gap (d) in this example is near-optimal for maximizing the size of the 800 $\mathrm{V} / \mathrm{cm}$ region, given a $400 \mathrm{~V}$ pulse, according to Figure $4 \mathrm{a}$.

The pair of needle electrodes in Figure 2e can be similarly analyzed. In Figure $2 \mathrm{e}, d / D=10$ and $V / D=10 \mathrm{kV} / \mathrm{cm}$. As-

(a)

(b) (c)
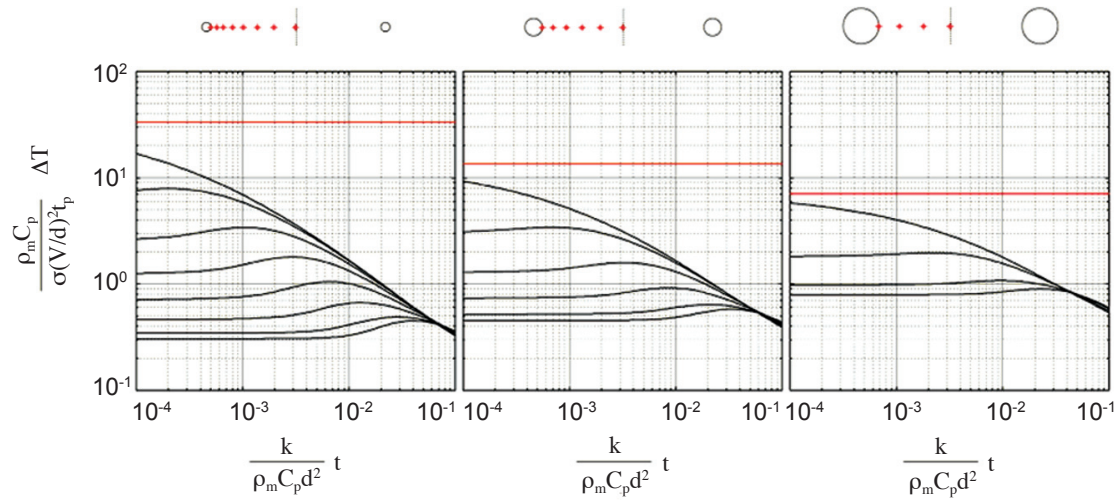

Figure 6: Dissipation of heat generated by a single pulse between two parallel needle electrodes. Graphs show the evolution of temperature with time at various points along the centerline between electrode centers. Temperature rise $(\Delta \mathrm{T})$ and time $(\mathrm{t})$ are given in a dimensionless form to allow general use. Asterisks in above electrode depictions mark exact locations from where the temperature is reported in the several plots in each pane. In each case, the highest and lowest curves correspond to the electrode inner surface and midpoint between electrodes, respectively. Eight curves in (a) are for $d / D=20$, six curves in $(\mathbf{b})$ are for $d / D=10$, and four curves in (c) are for $d / D=5$. Exact positions of marked points can be found by starting with the point on the inner electrode surface (D/2 from electrode center) and marking subsequent points at intervals of $a^{\mathrm{n}}(\mathrm{D} / 2)$, where this factor is 1.252 for (a), 1.203 for (b), and 1.151 for (c). Top horizontal line designates the maximal temperature rise within the object just at the end of the pulse. suming the same target $800 \mathrm{~V} / \mathrm{cm}$ electric field strength, locate the appropriate curve where $E /(V / D)=0.08$ or $10^{-1.1}$, which lies between the fourth and fifth curves from the top (10 $10^{-1.2}$ and $10^{-1.0}$, respectively). Thus, the appropriate coordinates on Figure $4 b$ would be $(x=10, y=30)$. The treatment area is then $30 D^{2}$, or $30 \mathrm{~mm}^{2}$.

The asterisks on each curve denote the point where the region containing electric field strengths above a certain level (different for each curve) splits in two. Since this point lies to the right of the asterisk, there are actually two $15 \mathrm{~mm}^{2}$ treated areas. This can be verified by seeing that the radius of the $800 \mathrm{~V} / \mathrm{cm}$ contour around each electrode in Figure 2e is about $2.2 \mathrm{~mm}$. To maximize the area with $800 \mathrm{~V} / \mathrm{cm}$ electric field strength, it can be inferred by tracing the curve of constant $E /(V / D)$ to the left, that the optimal $d / D$ ratio would be about 7 . This corresponds 
with an inter-electrode separation of $7 \mathrm{~mm}$, at which point the treated area would be about 50\% larger than area achieved with the $10 \mathrm{~mm}$ spacing shown in Figure $2 \mathrm{e}$.

For this figure, areas and volumes with electric field strengths greater than a particular value were computed numerically by integrating $E>$ cutoff across the entire tissue, which produces an area or volume since this Boolean expression is either 1 or 0 at all points. One minor comment is that it was necessary to multiply this integrand by $2 \pi r$ to obtain treated volumes for the disk electrodes case, where (r) is the radial distance. It is important to remember when using needle electrodes that the treated area along various planes perpendicular to the electrodes will vary, with the largest regions of any particular electric field strength existing on the midplane (L/2); except for some edge effects at the distal and proximal ends of the electrode active surfaces, which may increase the size of some of the higher electric field strength regions locally. This manifests as a somewhat further extent of the treated region in the space peripheral to the electrodes than would be indicated from Figure 4b, an effect that becomes more prominent as $L / d$ decreases. Note also that the treated area begins to separate along the electrode-electrode midline from the top and bottom towards the center as $d / D$ is increased beyond the optimal values from Figure $4 \mathrm{~b}$. Refer to the $400 \mathrm{~V} / \mathrm{cm}$ surface in the $3 \mathrm{D}$ pane of Figure $4 \mathrm{~b}$ for an example.

Table II examines this discrepancy between the treatment volume predicted for the case of $1 \mathrm{~mm}$ diameter parallel needle electrodes by the 2D model and that for the full 3D model, where the three columns correspond to the circles marked along the curve where $E /(V / D)=0.1$ in Figure $4 \mathrm{~b}$. From these data, which were computed for the same geometries as in Table I, it is clear that the 2D model is more accurate as $L$ is increased. Increasing $d$, however, has a more complex effect on this error; the $2 \mathrm{D}$ model at an inter-electrode gap of $7.5 \mathrm{~mm}$ is most accurate in this case but is least accurate for $d=10 \mathrm{~mm}$ (note that $d=7.5 \mathrm{~mm}$ is the point in Figure $4 \mathrm{~b}$ where the $2 \mathrm{D}$ treatment region splits in two). This can be understood by considering that the erosion of the treated region directly between the electrodes, above and below the midplane (L/2), offsets much of the additional volume in the spaces peripheral to the electrodes, so that it is not the actual shape of the treated region that is most accurate for $d=7.5 \mathrm{~mm}$, but only the total volume. The column where $d=5 \mathrm{~mm}$ is fairly accurate in both the total volume and the shape of the treated region, so that the $2 \mathrm{D}$ model is in fact most useful to determine treatment volume when $L / d$ is large and where $d / D$ is below the point at which the treatment region splits. Note also that the case where $L$ and $d$ are both 10 $\mathrm{mm}$ matches the $100 \mathrm{~V} / \mathrm{cm}$ surface depicted in Figure $1 \mathrm{~b}$.

\section{The Effect of Tissue Heterogeneity}

In a treatment, if the impedance distribution in the targeted region is homogenous, the results in Figure 4 can be applied directly to estimate the size of the treated region as a function of electrode geometry and voltage applied. However, there can be factors that would make the targeted domain heterogeneous, such as the presence of large blood vessels, multiple tissue types, or tissues with anisotropic properties, such as muscle. Figure 5 is an example of how tissue heterogeneity can affect the electric field distribution. Under these circumstances, the user would need to use our guidelines to make their own model including this heterogeneous conductivity and tailor our guidelines to their specific procedure.

Figure 5 provides three essential examples of how local heterogeneities in tissue conductivity affect the electric fields that develop within the nearby tissue. The first graph (Fig. 5a) shows a 5-mm diameter sphere when one fifth background conductivity is present between the electrodes. As a result of the higher impedance path for current traveling through the inclusion along the electrode-electrode axis, the current density directly between the electrode surfaces to the left and right sides of the low conductivity sphere, has fallen substantially. This has caused the $800 \mathrm{~V} / \mathrm{cm}$ surface surrounding the electrodes to become locally smaller. The electric potential in this space is, therefore, much closer to the nearby electrode voltage and as a result will cause other regions of tissue to experience a higher than normal electric field as the electric potential establishes continuity. This is evident based on the appearance of a separate $800 \mathrm{~V} / \mathrm{cm}$ surface within the inclusion with an hourglass shape. At this location, the electric potential will traverse the extra voltage necessary to connect the opposite regions that were varying more gradually than normal. In this particular case, the higher electric field strength corresponds with a focusing conical flow of a relatively small amount of current into the surface of the inclusion from one side and leaving in a similar fashion from the opposite side, where the net current flow is in the direction of the electrode-electrode axis.

The second graph (Fig. 5b) reveals the effect when the inclusion is five times more conductive than the surrounding tissue. In this figure, the path impedance between the electrodes and through the inclusion is substantially less than normal, leading to elevated electric field strengths in the spaces between each electrode and the inclusion. This is evident from the extension of the $800 \mathrm{~V} / \mathrm{cm}$ and $1600 \mathrm{~V} / \mathrm{cm}$ surfaces to the inclusion surface. In these two regions, the effect is that the electric potential is further removed from the electrode voltages than normal. This will cause the electric potential to vary gradually over the high conductivity inclusion in order to monotonically connect the two regions at opposite ends. The result is that the electric field strength inside the inclusion (and extending above and below its center) will become smaller than normal, a fact that is illustrated by the appearance of a hole in the $400 \mathrm{~V} / \mathrm{cm}$ region that is centered 
within the high conductivity inclusion. The mechanism for the difference in this case is a shunting of extra current from surrounding normal tissue into one hemisphere and out of the other, where net current of course travels along the electrodeelectrode axis. However, relatively little voltage drop will occur within the inclusion since it has a five-fold elevated conductivity and the extra current is not sufficient to offset this fact. Finally, the reason the hole extends into the normal tissue is that the high conductivity inclusion is providing a local short-cut for charges traveling near the inclusion surface but within the normal tissue.

The third graph (Fig. 5c) shows what will happen when a thin shell of low-conductivity tissue surrounds a core of highconductivity tissue, as is the case within regions bounded by an endothelium, such as blood vessels. The path impedance from one electrode to the other and through the center of the inclusion will be between that for the two previous cases, but is approximately equal to the case in Figure 1b, which can be seen by the nearly undisturbed shapes of the primary electric field surfaces. Though the electric potential and local current densities are relatively unchanged in the space between the electrodes and the inclusion boundaries, the core-shell structure has an interesting effect of focusing the electric field onto certain portions of the shell, while reducing it greatly within the core. If the two regions are quite different in terms of their conductivities, this can be understood by considering that the potential difference across the entire inclusion will be split almost entirely between the two primary locations where current either enters or exits the core through the shell. In Figure 5c, this can be observed as the opposite halos of $1600 \mathrm{~V} / \mathrm{cm}$ electric fields that correspond with conical influx or efflux of current from one electrode to the other and through the inclusion. Notice also that the hole in the 400 $\mathrm{V} / \mathrm{cm}$ surface is less pronounced than in Figure $5 \mathrm{~b}$, since the concentrated current visible as the $800 \mathrm{~V} / \mathrm{cm}$ and $1600 \mathrm{~V} / \mathrm{cm}$ extended surfaces in Figure $5 \mathrm{~b}$ is spread and greatly diminished by the low conductivity shell, and the hole does not protrude into normal tissue since the shell prevents the local short-cut discussed for Figure $5 b$.

The lessons from these three cases also apply in a general sense to any local perturbation in conductivity within a section of tissue where a macroscopic electric field has been imposed. Analogous to the electrode voltages are the bounding electric potentials, set up across the local control volume that includes the perturbation, and induced by the more global variation of impedance and ultimately the electrode voltages. In fact, the line of reasoning from Figure $5 \mathrm{c}$ can lead to an understanding of how local electric field strength translates into transmembrane potential. From the discussion of Figure 5c, one other important phenomenon becomes apparent. The high electric fields that will develop across the shell of such core-shell objects will cause electroporation locally long before it occurs in the surrounding tissues. This will provide an easier path for current to reach the high-conductivity core, leading to a much increased flow of current across the newly electroporated shell regions, and increasing somewhat the total amount of Joule heating. Depending on the details of the case, this could involve a pattern of microscopically focused large increases in temperature, visible as small paths of thermal damage across strategic points in the endothelium of large blood vessels or other lumen-filled cavities, a phenomenon that has been observed in recent animal studies of IRE (2) and may be the cause of the vascular lock observed even with presumably reversible pulses (32-34). Though not primarily responsible for the effectiveness of IRE, this phenomenon will enhance its therapeutic effect by causing tissues that survive the pulse itself to die later of local vascular occlusion (2).

Even with the large perturbations in conductivity from the three examples of Figure 5, neither the total power consumption (Fig. 5a-c drew $2.78 \mathrm{~kW}, 2.92 \mathrm{~kW}$, and $2.81 \mathrm{~kW}$ as compared with $2.83 \mathrm{~kW}$ for Figure $1 \mathrm{~b}$, respectively, for $\sigma=0.2$ $\mathrm{S} / \mathrm{m})$ nor the shapes of the lower electric field surfaces (100 $\mathrm{V} / \mathrm{cm}$ and $200 \mathrm{~V} / \mathrm{cm}$ ) has been affected significantly. This is primarily due to the fact that the inclusions are far from the electrode surfaces, where the electric potential changes most gradually and where perturbations will have only a local effect on electric fields. This is not the case when poor electrode-tissue contact is present. In fact, this can become a controlling influence on the strength of the electric fields that will develop within the tissue, and its effects can be understood most simply by assuming that the effective voltage across the electrodes will diminish to an amount approximately equal to $(\mathrm{K} / \sigma) /\left(2 \mathrm{z}_{\mathrm{e}} / \mathrm{A}_{\mathrm{e}}+\mathrm{K} / \sigma\right)$ times the applied voltage difference, where $z_{e}$ and $A_{e}$ are the electrode impedance (in $\Omega \mathrm{m}^{2}$ ) and area per electrode. This simplification makes the inherent assumption that current density is uniform across the electrode surfaces, but a more accurate prediction is possible through the incorporation into the numerical model of the complete electrode model (31), which connects the electrode voltage and impedance to the electric potential in the adjacent tissue through the normal current density across the electrode-tissue boundary as:

$$
V_{e}=\phi+z_{e} \sigma \frac{\partial \phi}{\partial n}
$$

The effect of electrode impedance will diminish for larger electrode surfaces and when $K$ is larger, but it will always be a source of some error and also of surface heating.

If there is concern that the impedance distribution of the tissue is not homogenous, the impedance distribution within the domain needs to be established to properly model and predict the area ablated using IRE. The user must make their own model as described in the methods section with an accurate knowledge of the distribution of $\sigma$ as well as the electrode 
configuration. An example of a method to obtain the anatomy of the targeted region is to use an imaging technique such as MRI or CT. Once the tissues in the targeted region are identified, their properties can be taken from literature (35) if the user cannot measure the properties directly.

\section{Change in Effective Conductivity of Tissue Due to Electroporation}

Researchers have shown that there is a change in tissue impedance during and as result of electroporation (13, 36-38). Even though such changes were not incorporated into our models, such changes would be important if the area is heterogeneous and especially for the needle configuration. These changes can be readily incorporated into the user's numerical models $(12,37)$. Many electroporation devices monitor the current being applied, which can be used to measure the bulk conductivity of the tissue as described in Figure 3. Monitoring the impedance change during IRE is an active means for the physician to monitor the procedure. Furthermore, the region that has been irreversibly electroporated can be imaged using electrical impedance tomography $(12,37)$, to verify that the targeted region has been successfully treated.

\section{Heat Dissipation After a Single Pulse}

If it is necessary to take into consideration the thermal effects from a treatment, then other tissue properties such as the mass density, heat capacity and thermal conductivity are needed. If these properties cannot be directly measured, the properties of the tissue can be taken from the literature, for example from (35). It should be noted that the thermal and electrical conductivities of biological tissues are dependent on temperature and their dependence can be found in literature and incorporated into the models if necessary. Since IRE produces negligible heating, the change in conductivity is not significant. For example, in liver the thermal and electrical conductivities vary by about $0.25 \%$ and $1.5 \%$ per degree Celsius, respectively (35). Properties for metabolism and blood flow can be found in (39) even though it has been suggested that these factors have a negligible contribution to the overall temperature distribution as compared with Joule heating (22). Futhermore, blood flow can be neglected as revealed by the results presented in (2) that showed that perfusion stops during such a procedure, which may assist in inducing total necrosis of the tissue.

Figure 6 was generated to enable the duration, extent and degree of the transient hyperthermia following an electroporation pulse, applied between needle electrodes in any uniform tissue, to be predicted based on approximate knowledge of tissue electrical and thermal properties and the details of the pulse.

The application of a short electroporation pulse across needle electrodes will cause a sudden deposition of a highly non-uniform amount of heat (2). Immediately after the pulse (and to some extent during the pulse), this heat will begin to diminish in strength as it spreads throughout the tissue as described by the heat diffusion equation. The initial heat deposition can be seen as the dimensionless temperature rise at each marked point in the tissue at the far left of the three graphs in Figure 6. This heating is so non-uniform that points far from the electrode surface are not subjected to the more intense heating near to the electrodes until some time has passed, as can be observed from the bump in temperature that occurs at progressively later times as one looks at points further from the electrode surface. By the time the temperatures have stabilized at the far right side of the Figure 6 plots, an additional pulse can be applied with minimal cumulative heating of the tissues.

As an example, consider a $100 \mu$ s pulse of $1000 \mathrm{~V}$ as depicted in Figure 2e that is applied across a tissue with $\rho=1050$ $\mathrm{kg} / \mathrm{m}^{3}, c_{p}=3600 \mathrm{~J} / \mathrm{kgK}, k=0.5 \mathrm{~W} / \mathrm{mK}$, and $\sigma=0.2 \mathrm{~S} / \mathrm{m}$. The time factor on the $\mathrm{x}$-axis of Figure 6 is then $0.00132 \mathrm{~s}^{-1}$, and the temperature factor on the $\mathrm{y}$-axis becomes $18.9 \mathrm{~K}^{-1}$. We can then predict the maximum temperature rise, which occurs at the inner surface of the electrode just at the end of the pulse, by reading the height of the horizontal line in the appropriate graph (Fig. 6b), approximately 13 . So, the temperature rise would be $13 /\left(18.9 \mathrm{~K}^{-1}\right)$, or about $0.7 \mathrm{~K}$. After 1 second ( 0.00132 on the $\mathrm{x}$-axis), the temperature at this point (upper curve) will have fallen to $0.25 \mathrm{~K}$, and the temperature at the middle of the tissue (lowest curve) peaks at $0.03 \mathrm{~K}$, a full 30 seconds after the pulse $\left(0.04 / 0.00132 \mathrm{~s}^{-1}\right)$. This pulse then is essentially non-thermal and could be applied at $1 \mathrm{~Hz}$ tens of times without causing thermal damage.

In the full 3D case, things would be somewhat different. It is clear that the amount of heat deposition will be higher than expected, which has already been demonstrated in the discussion of Figure $3 \mathrm{~b}$, and that this additional heating will be deposited primarily in the space peripheral to the electrodes (as can be seen from Fig. 1b) and locally at the electrode tip and junction with the shaft. But at the same time, this heat will be provided with a greater volume of cool tissue into which to spread. The result is that the most long-lived region of hyperthermia will be in the space between the centers of the electrodes and midway along their active lengths, where the $2 \mathrm{D}$ model is most accurate. To design pulse parameters to avoid thermal damage, the data in Figure 6 are, thus, sufficient for treatment planning in most cases.

For the case of disk electrodes, the heating is much more uniform. Aside from the pair of rings with high electric field strengths (for example see the $1600 \mathrm{~V} / \mathrm{cm}$ regions in Fig. 2c), the variation of electric potential in the space directly between the electrodes and from one electrode surface to the other is quite linear, so the Joule heating will be much less heteroge- 
neous than was the case for needle electrodes. An unfortunate consequence of this is that the heat will take longer to diffuse from the treated tissue, so the best practice would be to limit the total input of heat over the course of several pulses and not to depend on heat diffusion or on blood flow to withdraw heat [the best possibility for removal of heat would be with the use of cooled electrodes (23)]. As a rule of thumb, the best practice would be to compute the power consumption from the cell constant in Figure 3a, then assume that all of this energy (power multiplied by pulse duration) is deposited within the cylinder of tissue directly between the electrodes, of diameter $D$, which will predict a bulk temperature rise according to the electrical and thermal properties of the tissue.

A convenient equation to estimate the increase in temperature for the plate configuration from the Joule heating is:

$$
\Delta T=\frac{\sigma}{\rho c_{p}}|\nabla \phi|^{2} \Delta t,
$$

where $\Delta t$ is the total duration of the pulses. This equation assumes no heat dissipation between the pulses, and no fringe effects at the electrode edge. Furthermore, this equation assumes that the biological properties are uniform and the contributions from blood flow, metabolic heat, and electrode heat dissipation are negligible.

\section{Thermal Dose Assessment and Measuring Damage}

One of the distinguishing features of irreversible electroporation is that it does not induce thermal damage $(40,41)$. To assess whether a particular set of voltage parameters will induce thermal effects in addition to irreversible electroporation, the thermal damage can be calculated. Thermal damage, $\Omega$, is a timedependent process described by an Arrhenius type equation:

$$
\Omega=\int \xi e^{-E_{a} / R T} d t
$$

where $\xi$ is the frequency factor, $E_{a}$ is the activation energy, and $R$ is the universal gas constant (42-44). If the period of exposure is long, thermal damage can occur at temperatures as low as $42{ }^{\circ} \mathrm{C}$. However, $50{ }^{\circ} \mathrm{C}$ is generally chosen as the target temperature (43).

For procedures involving time varying temperatures, thermal damage can be assessed by calculating the amount of time it would take to equivalently damage the tissue as if it was held at a constant temperature, typically $43{ }^{\circ} \mathrm{C}(45)$. The following expression is the duration necessary to hold the tissue at $43{ }^{\circ} \mathrm{C}$ to result in an equivalent thermal dose:

$$
t_{43}=\sum_{t=0}^{t=f i n a l} R^{\left(43-T_{t}\right) \Delta t}
$$

where $T_{t}$ is the average temperature during $\Delta t$ with $R=0.25$ when $T_{t} \leq 43{ }^{\circ} \mathrm{C}$ and $R=0.5$ when $T_{t}>43{ }^{\circ} \mathrm{C}(46,47)$.

\section{Conclusion}

The goal of this work was to demonstrate the benefits of numerical models in designing protocols for irreversible electroporation surgery. The models provided can be used for guidance in the design of IRE protocols, and instructions with examples were also given on the creation of models for a custom treatment. Since IRE is a new technique to ablate undesirable tissue, the voltage parameters, specifically the electric field, to induce IRE in specific tissues is currently an active area of research. Our results demonstrate that the two most important things to consider when predicting the electric field distribution for an IRE treatment are the tissue conductivity distribution and the electrode configuration.

The figures present the influence of critical parameters, such as electrode size and shape of electrodes and tissue heterogeneity on the resulting electric field distribution. The figures are presented in a non-dimensional format, so the reader can directly plug their scenario specific pulse and geometry parameters into the plots to ensure the entire targeted area is above the electric field threshold to induce irreversible electroporation. If information is available with regards to the properties of the tissue being treated, information (such as thermal dissipation) can be approximated using the non-dimensional curves. We illustrate how the conductivity distribution, which may be due to the presence of large blood vessels, affects the electric field. Knowledge of the tissue properties and considering obstructions that might make the electric field distribution perturbed is important. Any perturbation can and must be incorporated into the models if known beforehand.

Although the IRE procedure is easy to perform, many surgical conditions are important to consider in the design and planning of IRE protocols. For example, the electric pulses can induce instantaneous muscle contraction. In this case, the surgeon may want to inject a muscle relaxant. While actually performing IRE, appropriate safety and preparation precautions must be taken. For example, needle and plate electrodes are typically used in conjunction with a conductive paste to ensure good electrical contact. Finally, the use of high voltage equipment is always a safety concern.

Studies have shown that the extent of electroporation can be imaged with MRI (27) and in real-time with electrical impedance tomography $(12,37)$. Irreversible electroporation has the advantages of being a tissue ablation technique that does not require adjuvant chemicals, it is easy, and has the capability of being monitored and controlled with diagnostic imaging. Furthermore, this study shows that the results from IRE surgery can be predicted beforehand through modeling to optimize the treatment. 


\section{References}

1. Davalos, R. V., Mir, L. M., and Rubinsky, B. Tissue ablation with irreversible electroporation. Annals of Biomedical Engineering 33, 223-231 (2005).

2. Edd, J., et al. In vivo results of a new focal tissue ablation technique: irreversible electroporation. IEEE Transactions on Biomedical Engineering 53, 1409-1415 (2006).

3. Rubinsky, B., Onik, G., and Mikus, P. Irreversible electroporation: A new ablation modality - clinical implications. Technology in Cancer Research and Treatment. 6, 37-48 (2007).

4. Weaver, J. C. and Chizmadzhev, Y. A. Theory of electroporation: a review. Bioelectrochem Bioenerg 41, 135-160 (1996).

5. Weaver, J. C. Electroporation of Biological Membranes from Multicellular to Nano Scales. IEEE Transactions on Dielectrics and Electrical Insulation 10, 754-768 (2003).

6. Sale, A. J. and Hamilton, W. A. Effects of high electric fields on micro-organisms. 1. Killing of bacteria and yeasts. Biochimica et Biophysica Acta 148, 781-788 (1967).

7. DeBruin, K. A. and Krassowska, W. Modeling electroporation in a single cell I. Effects of field strength and rest potential. Biophysical Journal 77, 1213-1224 (1999).

8. Davalos, R., Rubinsky, B., and Huang, Y. Electroporation: bio-electrochemical mass transfer at the nano Scale. Microscale Thermophysical Engineering 4, 147-159 (2000).

9. Diaz-Rivera, R. E. and Rubinsky, B. Electrical and thermal characterization of nanochannels between a cell and a silicon based micropore. Biomed Microdevices 8, 25-34 (2006).

10. Khine, M., et al. A single cell electroporation chip. Lab Chip 5, 3843 (2005).

11. Gowrishankar, T. R. and Weaver, J. C. An approach to electrical modeling of single and multiple cells. Proc Natl Acad Sci USA 100, 32033208 (2003).

12. Davalos, R. V., Otten, D. M., and Rubinsky, B. A feasibility study for electrical impedance tomography as a means to monitor tissue electroporation for molecular medicine. IEEE Transactions on Biomedical Engineering 49, 400-403 (2002).

13. Pavlin, M. and Miklavcic, D. Effective Conductivity of a Suspension of Permeabilized Cells: A Theoretical Analysis. Biophysical Journal 85, 719-729 (2003).

14. Gehl, J., et al. In vivo electroporation of skeletal muscle: threshold, efficacy and relation to electric field distribution. Biochimica et Biophysica Acta (BBA) 1428, 223-240 (1999).

15. Okino, M. and Mohri, H. Effects of a high-voltage electrical impulse and an anticancer drug on in vivo growing tumors. Japanese Journal of Cancer Research 78, 1319-1321 (1987).

16. Mir, L. M., et al. Electrochemotherapy, a novel antitumor treatment: first clinical trial. C R Acad Sci Ser III 313, 613-618 (1991).

17. Mir, L. M., et al. Electrochemotherapy potentiation of antitumour effect of bleomycin by local electric pulses. European Journal of Cancer 27, 68-72 (1991).

18. Mir, L. M., Therapeutic perspectives of in vivo cell electropermeabilization. Bioelectrochemistry 53, 1-10 (2001).

19. Mir, L. M. and Orlowski, S. Mechanisms of electrochemotherapy. Advanced drug delivery reviews 35, 107-118 (1999).

20. Miklavcic, D., et al. The importance of electric field distribution for effective in vivo electroporation of tissues. Biophysical Journal 74, 2152-2158 (1998).

21. Miklavcic, D., et al. A validated model of in vivo electric field distribution in tissues for electrochemotherapy and for DNA electrotransfer for gene therapy. Biochimica et Biophysica Acta 1523, 73-83 (2000).

22. Davalos, R. V., Rubinsky, B., and Mir, L.M. Theoretical analysis of the thermal effects during in vivo tissue electroporation. Bioelectrochemistry 61, 99-107 (2003).

23. Becker, S. M. and Kuznetsov, A. V. Thermal Damage Reduction Associated with in Vivo Skin Electroporation: A Numerical Investigation Justifying Aggressive Pre-Cooling. International Journal of Heat and Mass Transfer 50, 105-116 (2007).

24. Becker, S. M. and Kuznetsov, A. V. Numerical modeling of in vivo plate electroporation thermal dose assessment. Journal of Biomechanical Engineering 128, 76-84 (2006).

25. Weaver, J. C., Vaughana, T. E., and Chizmadzhev, Y. Theory of electrical creation of aqueous pathways across skin transport barriers. $A d$ vanced Drug Delivery Reviews 35, 21-39 (1999).

26. Martin, G. T., Pliquett, U. F., and Weaver, J. C. Theoretical analysis of localized heating in human skin subjected to high voltage pulses. Bioelectrochemistry 57, 55-64 (2002).

27. Lee, R. C., Zhang, D., and Hannig, J. Biophysical Injury Mechanisms in Electrical Shock Trauma, in Ann Rev Biomed Eng, pp. 477-509. Eds., M. L. Yarmish, K. R. Diller, and M. Toner. Annual Review Press: Palo Alto (2000).

28. Weaver, J. C., Electroporation of cells and tissues. IEEE Transactions on Plasma Science 28, 24-33 (2000).

29. Nuccitelli, R., et al. Nanosecond pulsed electric fields cause melanomas to self-destruct. Biochemical and Biophysical Research Communications 343, 351-353 (2006).

30. Pennes, H. H. Analysis of tissue and arterial blood temperatures in the resting forearm. J Appl Physiol 1, 93-122 (1948).

31. Somersalo, E., Cheney, M., and Isaacson, D. Existence and uniqueness for electrode models for electric current computed tomography. Siam J Appl Math 52, 1023-40 (1992).

32. Ramirez, L. H., et al. Electrochemotherapy on liver tumours in rabbits. Br J Cancer 77, 2104-2111 (1998).

33 Gehl, J., Skovsgaard, T., and Mir, L. M. Vascular reactions to in vivo electroporation: characterization and consequences for drug and gene delivery. Biochimica et Biophysica Acta 1569, 51-58 (2002).

34. Sersa, G., et al. Tumour blood flow changes induced by application of electric pulses. Eur J Cancer 35, 672-677 (1999).

35. Duck, F. A. Physical Properties of Tissues: A Comprehensive Reference Book. San Diego: Academic Press (1990).

36. Bhatt, D. L., Gaylor, D. C., and Lee, R. C. Rhabdomyolysis due to pulses electric fields. Plast Reconstr Surg 86, 1-11 (1990).

37. Davalos, R. V., et al. Electrical impedance tomography for imaging tissue electroporation. IEEE Transactions on Biomedical Engineering 51, 761-767 (2004).

38. Miklavcic, D., et al. Sequential Finite Element Model of Tissue Electropermeabilisation. in Proceedings of the 26th Annual International Conference of the IEEE EMBS. San Francisco, CA (2004).

39. Deng, Z. S. and Liu, J. Blood perfusion-based model for characterizing the temperature fluctuations in living tissue. Phys A STAT Mech Appl 300, 521-530 (2001).

40. Lee, R. C. and Despa, F. Distinguishing Electroporation from Thermal Injuries in Electrical Shock by MR Imaging. in Engineering in Medicine and Biology 27th Annual Conference. Shanghai, China: IEEE (2005).

41. Tropea, B. I. and Lee, R. C. Thermal Injury Kinetics in Electrical Trauma. J Biomech Engr 114, 241-250 (1992).

42. Henriques, F. C. and Moritz, A. R. Studies in thermal injuries: the predictability and the significance of thermally induced rate processes leading to irreversible epidermal damage. Arch Pathol. 43 , 489-502 (1947)

43. Diller, K. R. Modeling of bioheat transfer processes at high and low temperatures, in Bioengineering heat transfer, pp. 157-357. Ed., Y. I. Choi. Academic Press, Inc: Boston (1992).

44. Rylander, M. N., et al. Optimizing HSP Expression in Prostate Cancer Laser Therapy Through Predictive Computational Models. Journal of Biomedical Optics 11, 04111131-16 (2005)

45. Becker, S. M. and Kuznetsoz, A.V. Numerical Modeling of In Vivo Plate Electroporation Thermal Dose Assessment. ASME J of Biomechanical Engineering 128, 76-84 (2006).

46. Damianou, C., Hynynen, K., and Fan, X. Application of the Thermal Dose Concept for Predicting the Necrosed Tissue Volume During Ultrasound Surgery. Ultrasonics Symposium (1993).

47. Sapareto, S. and Dewey, W. Thermal dose determination in cancer therapy. Int J Radiation Oncology Biol Phys 10, 787-800 (1984).

Received: April 2, 2007; Revised: June 8, 2007; Accepted: June 13, 2007 